\title{
The structure of fixed-point sets of Lipschitzian type semigroups
}

\author{
DR Sahu ${ }^{1 *}$, RP Agarwal $^{2}$ and Donal O'Regan ${ }^{3}$
}

\section{"Correspondence:}

drsahudr@gmail.com

'Department of Mathematics, Banaras Hindu University, Varanasi, 221005, India

Full list of author information is

available at the end of the article

\begin{abstract}
The purpose of this paper is to establish some results on the structure of fixed point sets for one-parameter semigroups of nonlinear mappings which are not necessarily Lipschitzian in Banach spaces. Our results improve several known existence and convergence fixed point theorems for semigroups which are not necessarily Lipschitzian.

MSC: Primary 47H09; 47H10; secondary 47B20; 54C15

Keywords: asymptotic center; normal structure coefficient; pseudo-contractive semigroup; sunny nonexpansive retraction; uniformly convex Banach space; uniformly Lipschitzian semigroup; variational inequality
\end{abstract}

\section{Introduction}

Let $C$ be a nonempty subset of a Banach space $X$ and $T: C \rightarrow C$ a mapping. We use Fix $(T)$ to denote the set of all fixed points of $T$. A nonempty closed convex subset $D$ of $C$ is said to satisfy property $(\omega)$ with respect to mapping $T$ [1] if

$(\omega) \omega_{T}(x) \subset D$ for every $x \in D$,

where $\omega_{T}(x)$ denotes the set of all weak subsequential limits of $\left\{T^{n} x: n \in \mathbb{N}\right\}$. Moreover, $T$ is said to satisfy the $(\omega)$-fixed point property if $T$ has a fixed point in every nonempty closed convex subset $D$ of $C$ which satisfies property $(\omega)$. For a Lipschitzian mapping $S: C \rightarrow C$, we use the symbol $\sigma(S)$ to denote the exact Lipschitz constant of $S$, i.e.,

$$
\sigma(S)=\inf \{k \in[0, \infty]:\|S x-S y\| \leq k\|x-y\| \text { for all } x, y \in C\} .
$$

A mapping $T: C \rightarrow C$ is said to be

(1) nonexpansive if $\sigma(T)=1$,

(2) asymptotically nonexpansive [2] if $\sigma\left(T^{n}\right) \geq 1$ for all $n \in \mathbb{N}$ and $\lim _{n \rightarrow \infty} \sigma\left(T^{n}\right)=1$,

(3) uniformly L-Lipschitzian if $\sigma\left(T^{n}\right)=L$ for all $n \in \mathbb{N}$ and for some $L \in(0, \infty)$.

In general, the fixed-point set of a nonexpansive mapping need not be convex and can be extremely irregular. Suppose that $C$ is a nonempty closed convex bounded subset of a Banach space $X$ and $T: C \rightarrow C$ is a nonexpansive mapping with $\operatorname{Fix}(T) \neq \emptyset$. Obviously, $\operatorname{Fix}(T)$ is a closed set. Fix $(T)$ is convex if $X$ is strictly convex (see [3, 4]).

Nonexpansive retracts have been studied in several contexts (for example, convex geometry [5], extension problems [6], fixed point theory [7], optimal sets [8]). It is well known 
that if $C$ is a nonempty closed convex bounded subset of a Banach space and if a nonexpansive mapping $T: C \rightarrow C$ has a fixed point in every nonempty closed convex subset of $C$ which is invariant under $T$, then $\operatorname{Fix}(T)$ is a nonexpansive retract of $C$ (that is, there exists a nonexpansive mapping $R: C \rightarrow \operatorname{Fix}(T)$ such that $R_{\mid \operatorname{Fix}(T)}=I$ ) (see [7, Theorem 2]). The Bruck result was extended by Benavides and Ramirez [1] to the case of asymptotically nonexpansive mappings if the space $X$ was sufficiently regular.

The class of asymptotically nonexpansive mappings was introduced by Goebel and Kirk [2] in 1972 and they proved that if $C$ is a nonempty closed convex bounded subset of a uniformly convex Banach space, then every asymptotically nonexpansive self-mapping of $C$ has a fixed point. Several authors have studied the existence of fixed points of asymptotically nonexpansive mappings in Banach spaces having rich geometric structure, see [1, $9,10]$.

There is a class of mappings which lies strictly between the class of contraction mappings and the class of nonexpansive mappings. The class of pointwise contractions was introduced in Belluce and Kirk [11], and later it was called 'generalized contractions' in [12]. Banach's celebrated contraction principle was extended to this larger class of mappings as follows:

Theorem 1.1 [11,12] Let $C$ be a nonempty weakly compact convex subset of a Banach space and $T: C \rightarrow C$ a pointwise contraction. Then $T$ has a unique fixed point $x^{*}$, and $\left\{T^{n} x\right\}$ converges strongly to $x^{*}$ for each $x \in C$.

Kirk [13] combined the ideas of pointwise contraction [11] and asymptotic contraction [14] and introduced the concept of an asymptotic pointwise contraction. He announced that an asymptotic pointwise contraction defined on a closed convex bounded subset of a super-reflexive Banach space has a fixed point. Recently, Kirk and Xu [15] gave a simple and elementary proof of the fact that an asymptotic pointwise contraction defined on a weakly compact convex set always has a unique fixed point (with convergence of Picard iterates). They also introduced the concept of pointwise asymptotically nonexpansive mapping and proved that every pointwise asymptotically nonexpansive mapping defined on a closed convex bounded subset of a uniformly convex Banach space has a fixed point.

Every asymptotically nonexpansive mapping is uniformly $L$-Lipschitzian, and the $(\omega)$ fixed point property of uniformly $L$-Lipschitzian mappings is closely related to the class of nonexpansive and asymptotically nonexpansive mappings. In this connection, a deep result of Casini and Maluta [16] was generalized by Lim and $\mathrm{Xu}$ [17] as follows:

Theorem LX (Lim and Xu [17, Theorem 1]) Let X be a Banach space with a uniform normal structure and let $N(X)$ be the normal structure coefficient of $X$. Let $C$ be a nonempty bounded subset of $X$ and $T: C \rightarrow C$ a uniformly L-Lipschitzian mapping with $L<\sqrt{N(X)}$. Then $T$ satisfies the $(\omega)$-fixed point property.

The $(\omega)$-fixed point property plays a key role in the existence and approximation of solutions of fixed point problems and variational inequality problems, see [17-20].

The mapping theory for accretive mappings is closely related to the fixed point theory of pseudo-contractive mappings. Recently, applications of the semigroup result on the existence of solutions to certain partial differential equations have been explored in Hester and Morales [21]. They proved that the semigroup result directly implies the existence of 
unique global solutions to time evolution equations of the form $u^{\prime}=A u$, where $A$ is a combination of derivatives. In many applications, semigroups are not necessarily Lipschitzian. It is an interesting problem to extend fixed point existence results, namely Theorem LX, for semigroups of nonlinear mappings which are not necessarily Lipschitzian.

Motivated by the results above, in this paper we establish some results on the structure of fixed point sets for one-parameter semigroups of nonlinear mappings which are not necessarily Lipschitzian in Banach spaces. Our theorems significantly extend Theorem LX to more general Banach spaces and to a more general class of operators. We obtain a general convergence theorem for semigroups of non-Lipschitzian pseudo-contractive mappings. Our results improve several known fixed point problems and variational inequality problems for semigroups which are not necessarily Lipschitzian.

\section{Preliminaries}

Let $\mathbb{R}^{+}$denote the set of nonnegative real numbers, and let $\mathbb{N}_{0}$ denote the set of nonnegative integers. Throughout this paper, $G$ denotes an unbounded set of $\mathbb{R}^{+}:=[0, \infty)$ such that $s+t \in G$ for all $s, t \in G$ and $s-t \in G$ for all $s, t \in G$ with $s \geq t$ (often $G=\mathbb{N}_{0}$ or $\mathbb{R}^{+}$).

\subsection{Lipschitzian type mappings}

Let $C$ be a nonempty subset of a Banach space $X$ and $T: C \rightarrow C$ a mapping. Then $T$ is called

(i) pointwise contractive [11] if there exists a function $\alpha: C \rightarrow[0,1)$ such that $\|T x-T y\| \leq \alpha(x)\|x-y\|$ for all $x, y \in C ;$

(ii) asymptotic pointwise contractive [13] if for each $n \in \mathbb{N}$, there exists a function $\alpha_{n}: C \rightarrow[0,1)$ such that $\left\|T^{n} x-T^{n} y\right\| \leq \alpha_{n}(x)\|x-y\|$ for all $x, y \in C$, where $\alpha_{n} \rightarrow \alpha$ pointwise on $C$;

(iii) pointwise asymptotically nonexpansive [15] if for each integer $n \in \mathbb{N}$, $\left\|T^{n} x-T^{n} y\right\| \leq \alpha_{n}(x)\|x-y\|$ for all $x, y \in C$, where $\alpha_{n} \rightarrow 1$ pointwise;

(iv) asymptotically nonexpansive in the intermediate sense [22] provided $T$ is uniformly continuous and

$$
\limsup _{n \rightarrow \infty} \sup _{x, y \in C}\left(\left\|T^{n} x-T^{n} y\right\|-\|x-y\|\right) \leq 0
$$

(v) mapping of asymptotically nonexpansive type [23] if

$$
\limsup _{n \rightarrow \infty} \sup _{y \in C}\left(\left\|T^{n} x-T^{n} y\right\|-\|x-y\|\right) \leq 0 \quad \text { for all } x \in C
$$

Fix a sequence $\left\{a_{n}\right\}$ in $[0, \infty)$ with $a_{n} \rightarrow 0$. A mapping $T: C \rightarrow C$ is said to be nearly Lipschitzian with respect to $\left\{a_{n}\right\}$ [24] if for each $n \in \mathbb{N}$, there exists a constant $k_{n}>0$ such that

$$
\left\|T^{n} x-T^{n} y\right\| \leq k_{n}\left(\|x-y\|+a_{n}\right)
$$

for all $x, y \in C$. The infimum of constants $k_{n}$ in (2.2) is called nearly Lipschitz constant and is denoted by $\eta\left(T^{n}\right)$. A nearly Lipschitzian mapping $T$ with the sequence $\left\{\left(a_{n}, \eta\left(T^{n}\right)\right)\right\}$ is called 
(i) nearly contractive if $\eta\left(T^{n}\right)<1$ for all $n \in \mathbb{N}$,

(ii) nearly uniformly L-Lipschitzian if $\eta\left(T^{n}\right) \leq L$ for all $n \in \mathbb{N}$,

(iii) nearly uniformly $k$-contractive if $\eta\left(T^{n}\right) \leq k<1$ for all $n \in \mathbb{N}$,

(iv) nearly nonexpansive if $\eta\left(T^{n}\right)=1$ for all $n \in \mathbb{N}$,

(v) nearly asymptotically nonexpansive if $\eta\left(T^{n}\right) \geq 1$ for all $n \in \mathbb{N}$ with

$$
\lim _{n \rightarrow \infty} \eta\left(T^{n}\right)=1 \text {. }
$$

The mapping $T$ is said to be demicontinuous if, whenever a sequence $\left\{x_{n}\right\}$ in $C$ converges strongly to $x \in C$, then $\left\{T x_{n}\right\}$ converges weakly to $T x$. The mapping $T$ is said to be weakly contractive if

$$
\|T x-T y\| \leq\|x-y\|-\psi(\|x-y\|) \quad \text { for all } x, y \in C,
$$

where $\psi:[0, \infty) \rightarrow[0, \infty)$ is a continuous and nondecreasing function such that $\psi(0)=0$, $\psi(t)>0$ for $t>0$ and $\lim _{t \rightarrow \infty} \psi(t)=\infty$.

Let $C$ be a convex subset of a Banach space $X$ and $D$ a nonempty subset of $C$. Then a continuous mapping $P$ from $C$ onto $D$ is called a retraction if $P x=x$ for all $x \in D$, i.e., $P^{2}=P$. A retraction $P$ is said to be sunny if $P(P x+t(x-P x))=P x$ for each $x \in C$ and $t \geq 0$ with $P x+t(x-P x) \in C$. If the sunny retraction $P$ is also nonexpansive, then $D$ is said to be a sunny nonexpansive retract of $C$.

In what follows, we shall make use of the following lemmas:

Lemma 2.1 [3] Let $C$ be a nonempty closed convex subset of a Banach space $X$ and $T: C \rightarrow$ $C$ a continuous strongly pseudo-contractive mapping. Then Thas a unique fixed point in $C$.

Lemma 2.2 (Goebel and Reich [4, Lemma 13.1]) Let $C$ be a convex subset of a smooth Banach space $X, D$ a nonempty subset of $C$ and $P$ a retraction from $C$ onto $D$. Then the following are equivalent:

(a) $P$ is sunny and nonexpansive.

(b) $\langle x-P x, J(z-P x)\rangle \leq 0$ for all $x \in C, z \in D$.

(c) $\langle x-y, J(P x-P y)\rangle \geq\|P x-P y\|^{2}$ for all $x, y \in C$.

\subsection{Semigroups}

Let $C$ be a nonempty subset of a Banach space $X$. The one-parameter family $\mathcal{F}:=\{T(t)$ : $t \in G\}$ is said to be a strongly continuous semigroup of mappings from $C$ into itself if

(I) $T(0) x=x$ for all $x \in C$;

(II) $T(s+t)=T(s) T(t)$ for all $s, t \in G$;

(III) for each $x \in C$, the mapping $T(\cdot) x$ from $G$ into $C$ is continuous.

We denote by $\operatorname{Fix}(\mathcal{F})$ the set of all common fixed points of $\mathcal{F}$, i.e., $\operatorname{Fix}(\mathcal{F}):=$ $\bigcap_{t \in G} \operatorname{Fix}(T(t))$. For a Lipschitzian semigroup $\mathcal{F}$, we write

$$
\sigma(\mathcal{F}):=\liminf _{t \rightarrow \infty} \sigma(T(t))
$$

If $\mathcal{F}$ satisfies (I)-(III) and

$$
\lim _{t \rightarrow \infty}\|T(t) x-T(s) T(t) x\|=0 \quad \text { for all } x \in C \text { and } s \in G,
$$


then $\mathcal{F}$ is called asymptotically regular on $C$. If $\mathcal{F}$ satisfies (I)-(III) and

$$
\lim _{t \rightarrow \infty}\left(\sup _{x \in \widetilde{C}}\|T(t) x-T(s) T(t) x\|\right)=0 \quad \text { for all } s>0 \text { and bounded } \widetilde{C} \subseteq C,
$$

then $\mathscr{F}$ is called uniformly asymptotically regular on $C$.

A Lipschitzian semigroup $\mathcal{F}$ is called a

(i) uniformly L-Lipschitzian semigroup if $\sup _{t \in G} \sigma(T(t))=L<\infty$;

(ii) nonexpansive semigroup if $\sigma(T(t))=1$ for all $t \in G$;

(iii) asymptotically nonexpansive semigroup if $\sigma(T(t)) \geq 1$ for all $t \in G$ and $\lim _{t \rightarrow \infty} \sigma(T(t))=1$.

\subsection{Asymptotic center}

Throughout the paper, $(X,\|\cdot\|)$ is a Banach space which is assumed not to be Schur. That is, $X$ has weakly convergent sequences that are not norm convergent. Let $C$ be a nonempty closed convex subset of a Banach space $X$ and $\left\{x_{t}\right\}_{t \in G}$ a bounded set in $X$. Consider the functional $r_{a}\left(\cdot,\left\{x_{t}\right\}_{t \in G}\right): X \rightarrow \mathbb{R}^{+}$defined by

$$
r_{a}\left(x,\left\{x_{t}\right\}_{t \in G}\right)=\limsup _{G \ni t \rightarrow \infty}\left\|x_{t}-x\right\|, \quad x \in X .
$$

The infimum of $r_{a}\left(\cdot,\left\{x_{t}\right\}_{t \in G}\right)$ over $C$ is said to be the asymptotic radius of $\left\{x_{t}\right\}_{t \in G}$ with respect to $C$ and is denoted by $r_{a}\left(C,\left\{x_{t}\right\}_{t \in G}\right)$. A point $z \in C$ is said to be an asymptotic center of $\left\{x_{t}\right\}_{t \in G}$ with respect to $C$ if

$$
r_{a}\left(z,\left\{x_{t}\right\}_{t \in G}\right)=\inf \left\{r_{a}\left(x,\left\{x_{t}\right\}_{t \in G}\right): x \in C\right\} .
$$

The set of all asymptotic centers of $\left\{x_{t}\right\}_{t \in G}$ with respect to $C$ is denoted by $\mathcal{Z}_{a}\left(C,\left\{x_{t}\right\}_{t \in G}\right)$. A number $\operatorname{diam}_{a}\left(\left\{x_{t}\right\}_{t \in G}\right)=\lim \sup _{G \ni k \rightarrow \infty}\left(\sup \left\{\left\|x_{s}-x_{t}\right\|: s, t \geq k\right\}\right)$ is called an asymptotic diameter of $\left\{x_{t}\right\}_{t \in G}$. It is well known that if $X$ is reflexive, then $\mathcal{Z}_{a}\left(C,\left\{x_{t}\right\}_{t \in G}\right)$ is nonempty closed convex and bounded, and if $X$ is uniformly convex, then $\mathcal{Z}_{a}\left(C,\left\{x_{t}\right\}_{t \in G}\right)$ consists only of a single point, $\{z\}=\mathcal{Z}_{a}\left(C,\left\{x_{t}\right\}_{t \in G}\right)$, i.e., $z \in C$ is the unique point which minimizes the functional

$$
\limsup _{G \ni t \rightarrow \infty}\left\|x_{t}-x\right\|
$$

over $x \in C$.

\subsection{Normal structure}

Normal structure plays a key role in some problems of metric fixed point theory. Let $C$ be a nonempty bounded subset of a Banach space $X$. We denote by

$$
\operatorname{diam}(C)=\sup _{x, y \in C}\|x-y\|,
$$

the diameter of $C$. Put

$$
r_{C}(C)=\inf _{x \in C}\left\{\sup _{y \in C}\|x-y\|\right\} .
$$


This nonnegative real number is called the Chebyshev radius of $C$ relative to itself. The normal structure coefficient $N(X)$ of a Banach space $X$ is defined [25] by

$$
\begin{aligned}
N(X)= & \inf \left\{\frac{\operatorname{diam}(C)}{r_{C}(C)}: C\right. \text { is nonempty bounded convex subset } \\
& \text { of } X \text { with } \operatorname{diam}(C)>0\} .
\end{aligned}
$$

The space $X$ is said to have the uniformly normal structure if $N(X)>1$. It is well known that, for every uniformly convex Banach space $X, N(X)>1$. A weakly convergent sequence coefficient of $X$ is defined (see [25]) by

$$
\mathrm{WCS}(X)=\sup \left\{k: k \limsup _{n \rightarrow \infty}\left\|x_{n}\right\|<\operatorname{diam}_{a}\left(\left\{x_{n}\right\}\right) \text { for all }\left\{x_{n}\right\} \text { in } X \text { with } x_{n} \rightarrow 0\right\} \text {. }
$$

It is proved in [26, Theorem 1$]$ that

$$
\operatorname{WCS}(X)=\beta(X):=\inf \left\{D\left[\left\{x_{n}\right\}\right]: x_{n} \rightarrow 0,\left\|x_{n}\right\| \rightarrow 1\right\},
$$

where $D\left[\left\{x_{n}\right\}\right]:=\lim \sup _{m \rightarrow \infty}\left(\lim \sup _{n \rightarrow \infty}\left\|x_{m}-x_{n}\right\|\right)$. It is readily seen that

$$
1 \leq N(X) \leq \mathrm{WCS}(X) \leq 2
$$

The space $X$ is said to have the weak uniformly normal structure if $\operatorname{WCS}(X)>1$. If $X$ is a reflexive Banach space with modulus of convexity $\delta_{X}$, then

$$
\frac{1}{1-\delta_{X}(1)} \leq N(X) \leq \mathrm{WCS}(X)
$$

Thus, if $X$ is a uniformly convex Banach space, then $\operatorname{WCS}(X)>1$ and also the equation

$$
\frac{\alpha^{2}}{\operatorname{WCS}(X)} \delta_{X}^{-1}\left(1-\frac{1}{\alpha}\right)=1
$$

has a unique solution $\alpha>1$. A general formula for $\operatorname{WCS}(X)$ in an arbitrary Banach space is not known. In particular, it has been calculated that for a Hilbert space $H$,

$$
\operatorname{WCS}(H)=\sqrt{2} ;
$$

for $\ell_{p}(1 \leq p<\infty)$,

$$
\operatorname{WCS}\left(\ell_{p}\right)=2^{1 / p}
$$

and for $\ell_{\infty}$,

$$
\operatorname{WCS}\left(\ell_{\infty}\right)=1 .
$$


Remark 2.3 $\ell_{p}$ is an example of a reflexive Banach space such that $N(X)$ and $\mathrm{WCS}(X)$ are different. Indeed,

$$
N\left(\ell_{p}\right)=2^{(p-1) / p}<2^{1 / p}=\operatorname{WCS}\left(\ell_{p}\right), \quad \text { for } 1<p<2 .
$$

A Banach space $X$ is said to satisfy the Opial condition, if whenever a sequence $\left\{x_{n}\right\}$ in $X$ converges weakly to $x$, then

$$
\liminf _{n \rightarrow \infty}\left\|x_{n}-x\right\|<\liminf _{n \rightarrow \infty}\left\|x_{n}-y\right\| \quad \text { for all } y \in X \backslash\{x\} .
$$

The Opial modulus $r_{X}$ of $X$ is defined by

$$
r_{X}(c)=\inf \left\{\liminf _{n \rightarrow \infty}\left\|x_{n}+x\right\|-1\right\}
$$

where $c>0$ and the infimum is taken over all $x \in X$ with $\|x\| \geq c$ and all sequences $\left\{x_{n}\right\}$ in $X$ such that $w-\lim _{n \rightarrow \infty} x_{n}=0$ and $\liminf _{n \rightarrow \infty}\left\|x_{n}\right\| \geq 1$. For any Banach space $X$, we have the following inequality:

$$
1+r_{X}(1) \leq \mathrm{WCS}(X)
$$

\section{Nonemptiness of common fixed-point sets}

First, we introduce some wider classes of semigroups.

Definition 3.1 Let $C$ be a nonempty subset of a normed space $X$ and $\mathcal{F}:=\{T(t): t \in G\}$ a strongly continuous semigroup of mappings from $C$ into itself. The semigroup $\mathcal{F}$ is said to be nearly Lipschitzian if there exist a function $a(\cdot): G \rightarrow[0, \infty)$ with $\lim _{t \rightarrow \infty} a(t)=0$ and a function $\eta(\cdot): G \rightarrow(0, \infty)$ such that

$$
\|T(t) x-T(t) y\| \leq \eta(T(t))(\|x-y\|+a(t)) \quad \text { for all } x, y \in C \text { and } t \in G \text {. }
$$

For a nearly Lipschitzian semigroup $\mathcal{F}$, we write

$$
\eta(\mathcal{F}):=\liminf _{t \rightarrow \infty} \eta(T(t))
$$

We say $\mathcal{F}$ is

(a) pointwise nearly Lipschitzian if for each $t \in G$, there exist a function $a(\cdot): G \rightarrow[0, \infty)$ with $\lim _{t \rightarrow \infty} a(t)=0$ and a function $\alpha_{t}(\cdot): C \rightarrow(0, \infty)$ such that

$$
\|T(t) x-T(t) y\| \leq \alpha_{t}(x)(\|x-y\|+a(t)) \quad \text { for all } x, y \in C ;
$$

(b) pointwise nearly uniformly $\alpha(\cdot)$-Lipschitzian if there exist a function $a(\cdot): G \rightarrow[0, \infty)$ with $\lim _{t \rightarrow \infty} a(t)=0$ and a function $\alpha(\cdot): C \rightarrow(0, \infty)$ such that

$$
\|T(t) x-T(t) y\| \leq \alpha(x)(\|x-y\|+a(t)) \quad \text { for all } x, y \in C ;
$$


(c) asymptotic pointwise nearly Lipschitzian if for each $t \in G$, there exist a function $a(\cdot): G \rightarrow[0, \infty)$ with $\lim _{t \rightarrow \infty} a(t)=0$ and two functions $\alpha_{t}(\cdot), \alpha(\cdot): C \rightarrow(0, \infty)$ with $\lim _{t \rightarrow \infty} \alpha_{t}=\alpha$ pointwise such that

$$
\|T(t) x-T(t) y\| \leq \alpha_{t}(x)(\|x-y\|+a(t)) \quad \text { for all } x, y \in C
$$

We say that an asymptotic pointwise nearly Lipschitzian semigroup $\mathcal{F}$ is pointwise nearly asymptotically nonexpansive (pointwise asymptotically nonexpansive) if $\alpha_{t}(x) \geq 1$ for all $t \in G$ and $\lim _{t \rightarrow \infty} \alpha_{t}=1$ pointwise $\left(a(t)=0\right.$ and $\alpha_{t}(x) \geq 1$ for all $t \in G$ and $\alpha_{t}(x) \rightarrow 1$ pointwise). Further, we say that an asymptotic pointwise nearly Lipschitzian semigroup $\mathscr{F}$ is asymptotic pointwise nearly contractive if $\alpha_{t} \rightarrow \alpha$ pointwise and $\alpha(x) \leq k<1$ for all $x \in C$. The semigroup $\mathcal{F}$ is said to be nearly uniformly $L$-Lipschitzian if there exist a constant $L \in[0, \infty)$ and a function $a(\cdot): G \rightarrow[0, \infty)$ with $\lim _{t \rightarrow \infty} a(t)=0$ such that

$$
\|T(t) x-T(t) y\| \leq L(\|x-y\|+a(t)) \quad \text { for all } x, y \in C
$$

The nearly uniformly $L$-Lipschitzian semigroup will be called nearly nonexpansive semigroup.

Before presenting the main result of this section, we give another definition:

Definition 3.2 Let $C$ be a nonempty weakly compact convex subset of Banach space $X, \mathcal{F}:=\{T(t): t \in G\}$ a strongly continuous semigroup of mappings from $C$ into itself. A nonempty closed convex subset $D$ of $C$ is said to satisfy property $(\omega)$ with respect to semigroup $\mathcal{F}$ if

( $\omega) \omega_{\mathcal{F}}(x) \subset D$ for every $x \in D$,

where $\omega_{\mathcal{F}}(x)$ denotes the set of all weak limits of $\left\{T\left(t_{n}\right) x: n \in \mathbb{N}\right\}$ as $t_{n} \rightarrow \infty$.

The semigroup $\mathcal{F}$ is said to satisfy the $(\omega)$-fixed point property if $\mathcal{F}$ has a common fixed point in every nonempty closed convex subset $D$ of $C$ which satisfies property $(\omega)$.

We now establish that a semigroup $\mathcal{F}$ of a certain class of Lipschitzian type mappings satisfies the $(\omega)$-fixed point property.

Theorem 3.3 Let $X$ be a Banach space with weak uniformly normal structure, $C$ a nonempty weakly compact convex subset of $X$ and $\mathcal{F}=\{T(t): t \in G\}$ a strongly continuous semigroup of demicontinuous mappings from $C$ into itself. Suppose that for each $t \in G$, there exist a function $a(\cdot): G \rightarrow[0, \infty)$ with $\lim _{t \rightarrow \infty} a(t)=0$ and two functions $\alpha_{t}(\cdot), \alpha(\cdot): C \rightarrow(0, \infty)$ with $\lim _{t \rightarrow \infty} \alpha_{t}=\alpha$ pointwise and $\sup _{x \in C} \alpha(x)<\sqrt{\mathrm{WCS}(X)}$ such that

$$
\|T(t) x-T(t) y\| \leq \alpha_{t}(x)(\|x-y\|+a(t)) \quad \text { for all } x, y \in C
$$

Also suppose that there exists a nonempty closed convex subset $M$ of $C$ which satisfies property $(\omega)$ with respect to $\mathcal{F}$. Then:

(a) For arbitrary $x_{0} \in M$, there exist a sequence $\left\{t_{n}\right\}$ in $G$ with $\lim _{n \rightarrow \infty} t_{n}=\infty$ and an iterative sequence $\left\{x_{m}\right\}$ in $M$ defined by

$$
x_{m}=w-\lim _{n \rightarrow \infty} T\left(t_{n}\right) x_{m-1} \quad \text { for all } m \in \mathbb{N} .
$$


(b) If $\mathcal{F}$ is asymptotically regular on $C$, then there exists an element $x^{*} \in M \cap \operatorname{Fix}(\mathcal{F})$ such that $\left\{x_{m}\right\}$ converges strongly to $x^{*}$.

Proof

(a) Since one can easily construct a nonempty closed convex separable subset $C_{0}$ of $\mathrm{C}$ which is invariant under each $T(t)$ (i.e., $T(t)\left(C_{0}\right) \subset C_{0}$ for $t \in G$ ), we may assume that $C$ itself is separable.

The separability of $C_{0}$ makes it possible to select a sequence $\left\{T\left(t_{n}\right) x\right\}$ of $\{T(t) x\}_{t \in G}$ such that

$\left\{T\left(t_{n}\right) x\right\}$ is weakly convergent for every $x \in C$

and

$$
\lim _{n \rightarrow \infty} \alpha_{t_{n}}(x)=\alpha(x) \quad \text { for every } x \in C .
$$

For any $x_{0} \in M \subset C$, consider a sequence $\left\{T\left(t_{n}\right) x_{0}\right\}$ in $C$. Suppose $w-\lim _{n \rightarrow \infty} T\left(t_{n}\right) x_{0}=$ $x_{1} \in C$. Using property $(\omega)$, we obtain that $x_{1} \in M$. Now we can construct a sequence $\left\{x_{m}\right\}$ in $M$ in the following way:

$$
\left\{\begin{array}{l}
x_{0} \in M \quad \text { arbitrary, } \\
x_{m}=w-\lim _{n \rightarrow \infty} T\left(t_{n}\right) x_{m-1} \quad \text { for all } m \in \mathbb{N} .
\end{array}\right.
$$

(b) The weak asymptotic regularity of $\mathcal{F}$ ensures that $x_{m}=w-\lim _{n \rightarrow \infty} T\left(t_{n}+t_{r}\right) x_{m-1}$, $t_{r} \in G$. We now show that $\left\{x_{m}\right\}$ converges strongly to a common fixed point of $\mathcal{F}$. Set

$$
L:=\sup _{x \in C} \alpha(x), \quad D_{m}:=\limsup _{n \rightarrow \infty}\left\|x_{m}-T\left(t_{n}\right) x_{m}\right\|
$$

and

$$
R_{m}:=\limsup _{n \rightarrow \infty}\left\|x_{m+1}-T\left(t_{n}\right) x_{m}\right\|
$$

for all $m=0,1,2, \ldots$ By the property of $\operatorname{WCS}(X)$, we have

$$
R_{m}=\limsup _{n \rightarrow \infty}\left\|x_{m+1}-T\left(t_{n}\right) x_{m}\right\| \leq \frac{1}{\operatorname{WCS}(X)} D\left[\left\{T\left(t_{n}\right) x_{m}\right\}\right] .
$$

By the asymptotic regularity of $\mathcal{F}$ and the $w$-l.s.c. of the norm $\|\cdot\|$, we have

$$
\begin{aligned}
D_{m} & =\limsup _{r \rightarrow \infty}\left\|x_{m}-T\left(t_{r}\right) x_{m}\right\| \\
& \leq \limsup _{r \rightarrow \infty}\left(\limsup _{s \rightarrow \infty}\left\|T\left(t_{s}\right) x_{m-1}-T\left(t_{r}\right) x_{m}\right\|\right) \\
& \leq \limsup _{r \rightarrow \infty}\left(\limsup _{s \rightarrow \infty}\left(\left\|T\left(t_{s}\right) x_{m-1}-T\left(t_{r}+t_{s}\right) x_{m-1}\right\|+\left\|T\left(t_{r}+t_{s}\right) x_{m-1}-T\left(t_{r}\right) x_{m}\right\|\right)\right) \\
& \leq \limsup _{r \rightarrow \infty}\left(\limsup _{s \rightarrow \infty}\left(\alpha_{t_{r}}\left(x_{m}\right)\left(\left\|T\left(t_{s}\right) x_{m-1}-x_{m}\right\|+a\left(t_{r}\right)\right)\right)\right)
\end{aligned}
$$




$$
\begin{aligned}
& =\alpha\left(x_{m}\right) \limsup _{s \rightarrow \infty}\left\|T\left(t_{s}\right) x_{m-1}-x_{m}\right\| \\
& \leq L R_{m-1} .
\end{aligned}
$$

On the other hand, by the asymptotic regularity of $\mathcal{F}$, we have

$$
\begin{aligned}
D\left[\left\{T\left(t_{n}\right) x_{m}\right\}\right]= & \limsup _{n \rightarrow \infty}\left(\limsup _{r \rightarrow \infty}\left\|T\left(t_{n}\right) x_{m}-T\left(t_{r}\right) x_{m}\right\|\right) \\
\leq & \limsup _{n \rightarrow \infty}\left(\operatorname { l i m s u p } _ { r \rightarrow \infty } \left(\left\|T\left(t_{n}\right) x_{m}-T\left(t_{n}+t_{r}\right) x_{m}\right\|\right.\right. \\
& \left.\left.+\left\|T\left(t_{n}+t_{r}\right) x_{m}-T\left(t_{r}\right) x_{m}\right\|\right)\right) \\
\leq & \limsup _{n \rightarrow \infty}\left(\limsup _{r \rightarrow \infty}\left(\alpha_{t_{n}}\left(x_{m}\right)\left(\left\|x_{m}-T\left(t_{r}\right) x_{m}\right\|+a\left(t_{n}\right)\right)\right)\right) \\
= & L D_{m} \\
\leq & L^{2} R_{m-1} .
\end{aligned}
$$

Set $\lambda:=\frac{L^{2}}{\operatorname{WCS}(X)}<1$. From (3.2), we obtain

$$
R_{m} \leq \lambda R_{m-1} \leq \lambda^{2} R_{m-2} \leq \cdots \leq \lambda^{m} R_{0} \rightarrow 0 \quad \text { as } m \rightarrow \infty
$$

For any $m \in \mathbb{N}$, one can see that

$$
\begin{aligned}
\left\|x_{m+1}-x_{m}\right\| & \leq \limsup _{n \rightarrow \infty}\left(\left\|x_{m+1}-T\left(t_{n}\right) x_{m}\right\|+\left\|T\left(t_{n}\right) x_{m}-x_{m}\right\|\right) \\
& \leq R_{m}+D_{m} \\
& =R_{m}+L R_{m-1} \\
& \leq(\lambda+L) R_{m-1} \\
& \vdots \\
& \leq(\lambda+L) \lambda^{m-1} R_{0}
\end{aligned}
$$

so it follows that $\left\{x_{m}\right\}$ is a Cauchy sequence in $M$. Let $\lim _{m \rightarrow \infty} x_{m}=v \in M$. Observe that

$$
\begin{aligned}
\left\|v-T\left(t_{n}\right) v\right\| & \leq\left\|v-x_{m+1}\right\|+\left\|x_{m+1}-T\left(t_{n}\right) x_{m}\right\|+\left\|T\left(t_{n}\right) x_{m}-T\left(t_{n}\right) v\right\| \\
& \leq\left\|v-x_{m+1}\right\|+\left\|x_{m+1}-T\left(t_{n}\right) x_{m}\right\|+\alpha_{t_{n}}\left(x_{m}\right)\left(\left\|x_{m}-v\right\|+a\left(t_{n}\right)\right) .
\end{aligned}
$$

Taking the limit superior as $n \rightarrow \infty$ on both sides, we get

$$
\begin{aligned}
\limsup _{n \rightarrow \infty}\left\|v-T\left(t_{n}\right) v\right\| & \leq\left\|v-x_{m+1}\right\|+R_{m}+\alpha\left(x_{m}\right)\left\|x_{m}-v\right\| \\
& \leq\left\|v-x_{m+1}\right\|+R_{m}+L\left\|x_{m}-v\right\| \rightarrow 0 \quad \text { as } m \rightarrow \infty .
\end{aligned}
$$

Hence $\lim _{n \rightarrow \infty} T\left(t_{n}\right) v=v$. Let $t \in G$. Note $T\left(t_{n}\right) v \rightarrow v$, so it follows from the demicontinuity of $T(t)$ that $T(t) T\left(t_{n}\right) v \rightarrow T(t) v$. Observe that $T(t) T\left(t_{n}\right) v=T\left(t+t_{n}\right) v \rightarrow v$. 
By the uniqueness of the weak limit of $\left\{T(t) T\left(t_{n}\right) v\right\}_{n \in \mathbb{N}}$, we have $T(t) v=v$. Therefore, $v \in M \cap \operatorname{Fix}(\mathcal{F})$.

Theorem 3.3 generalizes the result due to Górnicki [27] in the context of the ( $\omega)$-fixed point property for a wider class of mappings. Theorem 3.3 also extends corresponding results of Sahu, Agarwal and O'Regan [18], Sahu, Liu and Kang [28] and Sahu, Petruşel and Yao [29] for asymptotic pointwise nearly Lipschitzian semigroups. As $N(X) \leq \mathrm{WCS}(X)$, and there are Banach spaces for which $N(X)=1$ while $\operatorname{WCS}(X)>1$, the following result is an improvement on Casini and Maluta [16] and Lim and Xu [17, Theorem 1].

Corollary 3.4 Let $X$ be a Banach space with weak uniformly normal structure, $C$ a nonempty weakly compact convex subset of $X$ and $\mathcal{F}=\{T(t): t \in G\}$ a strongly continuous semigroup of demicontinuous nearly uniformly $L$-Lipschitzian of mappings from $C$ into itself. Suppose that $L<\sqrt{\mathrm{WCS}(X)}$ and that there exists a nonempty closed convex subset $M$ of $C$ which satisfies property $(\omega)$ with respect to $\mathcal{F}$. Then:

(a) For arbitrary $x_{0} \in M$, there exist a sequence $\left\{t_{n}\right\}$ in $G$ with $\lim _{n \rightarrow \infty} t_{n}=\infty$ and an iterative sequence $\left\{x_{m}\right\}$ in $M$ defined by

$$
x_{m}=w-\lim _{n \rightarrow \infty} T\left(t_{n}\right) x_{m-1} \quad \text { for all } m \in \mathbb{N} .
$$

(b) If $\mathcal{F}$ is asymptotically regular on $C$, then there exists an element $x^{*} \in M \cap \operatorname{Fix}(\mathcal{F})$ such that $\left\{x_{m}\right\}$ converges strongly to $x^{*}$.

Corollary 3.5 Let $X$ be a Banach space with weak uniformly normal structure, $C$ a nonempty weakly compact convex subset of $X$ and $\mathcal{F}=\{T(t): t \in G\}$ a strongly continuous semigroup of demicontinuous nearly uniformly L-Lipschitzian asymptotically regular mappings from $C$ into itself such that $L<\sqrt{\mathrm{WCS}(X)}$. Then $\mathcal{F}$ has a common fixed point in $C$.

\section{Common fixed-point sets as Lipschitzian retracts}

Theorem 4.1 Let $X$ be a uniformly Banach space with the Opial condition, $C$ a nonempty closed convex bounded subset of $X$ and $\mathcal{F}=\{T(t): t \in G\}$ a strongly continuous semigroup of demicontinuous mappings from $C$ into itself. Suppose that there exists a function $a(\cdot)$ : $G \rightarrow[0, \infty)$ with $\lim _{t \rightarrow \infty} a(t)=0$ such that

$$
\|T(t) x-T(t) y\| \leq \eta(T(t))(\|x-y\|+a(t)) \quad \text { for all } x, y \in C,
$$

where $\eta(T(t)) \in(0, \infty)$ with $\eta(\mathcal{F}):=\liminf _{t \rightarrow \infty} \eta(T(t))<\sqrt{\mathrm{WCS}(X)}$. Also suppose that $\mathcal{F}$ is asymptotically regular on $C$. Then $\operatorname{Fix}(\mathcal{F}) \neq \emptyset$ and $\operatorname{Fix}(\mathcal{F})$ is $\eta(\mathcal{F})$-Lipschitzian retract of $C$.

Proof Using similar arguments as in the proof of Theorem 3.3(a), we may select a sequence $\left\{T\left(t_{n}\right) x\right\}$ of $\{T(t) x\}_{t \in G}$ such that $\lim _{n \rightarrow \infty} \eta\left(T\left(t_{n}\right)\right)=\eta(\mathcal{F})$ and

$\left\{T\left(t_{n}\right) x\right\}$ is weakly convergent for every $x \in C$. 
Let $A: C \rightarrow C$ denote a mapping which associates with a given $x \in C$ a unique $z \in$ $\mathcal{Z}_{a}\left(C,\{T(t) x\}_{t \in G}\right)$, that is, $z=A x$. Since $A x=w-\lim _{n \rightarrow \infty} T\left(t_{n}\right) x$ for all $x \in C$, it follows from the lower weak semi-continuity of the norm that

$$
\|A x-A y\| \leq \eta(\mathcal{F})\|x-y\| \quad \text { for all } x, y \in C,
$$

i.e., $A$ is $\eta(\mathcal{F})$-Lipschitzian mapping. It follows that $A$ is uniformly continuous.

For any $x=x_{0} \in C$, consider a sequence $\left\{T\left(t_{n}\right) x_{0}\right\}$ in $C$. Suppose $w-\lim _{n \rightarrow \infty} T\left(t_{n}\right) x_{0}=$ $x_{1} \in C$. Now we can construct a sequence $\left\{x_{m}\right\}$ in $C$ in the following way:

$$
\left\{\begin{array}{l}
x=x_{0} \in C \quad \text { arbitrary, } \\
x_{m}=w-\lim _{n \rightarrow \infty} T\left(t_{n}\right) x_{m-1} \quad \text { for all } m \in \mathbb{N} .
\end{array}\right.
$$

From (4.1), we have

$$
x_{m+1}=A x_{m} \quad \text { for all } m \in \mathbb{N}_{0} .
$$

Set $L:=\eta(\mathcal{F}), \quad \lambda:=\frac{L^{2}}{\operatorname{WCS}(X)}<1, \quad D_{m}:=\lim \sup _{n \rightarrow \infty}\left\|x_{m}-T\left(t_{n}\right) x_{m}\right\|$ and $R_{m}:=$ $\limsup \sup _{n \rightarrow \infty}\left\|x_{m+1}-T\left(t_{n}\right) x_{m}\right\|$ for all $m=0,1,2, \ldots$. From (3.3), we have

$$
\begin{aligned}
\left\|A^{m+1} x-A^{m} x\right\| & =\left\|x_{m+1}-x_{m}\right\| \leq(\lambda+L) \lambda^{m-1} R_{0} \\
& \leq(\lambda+L) \lambda^{m-1} \operatorname{diam}(C)
\end{aligned}
$$

for $x \in C$ and $m \in \mathbb{N}$. It follows that

$$
\sum_{m=1}^{\infty} \sup _{x \in C}\left\|A^{m+1} x-A^{m} x\right\|<\infty
$$

Thus, the sequence $\left\{A^{m} x\right\}$ converges uniformly to a function $Q$ defined by

$$
Q x=\lim _{m \rightarrow \infty} A^{m} x \quad \text { for all } x \in C .
$$

For $x=x_{0}$, we have

$$
\begin{aligned}
\left\|Q x-T\left(t_{n}\right) Q x\right\| & \leq\left\|Q x-x_{m}\right\|+\left\|x_{m}-T\left(t_{n}\right) x_{m}\right\|+\left\|T\left(t_{n}\right) x_{m}-T\left(t_{n}\right) Q x\right\| \\
& \leq\left\|Q x-x_{m}\right\|+\left\|x_{m}-T\left(t_{n}\right) x_{m}\right\|+\eta\left(T\left(t_{n}\right)\right)\left(\left\|x_{m}-Q x\right\|+a\left(t_{n}\right)\right) .
\end{aligned}
$$

Taking the limit superior as $n \rightarrow \infty$, we get

$$
\limsup _{n \rightarrow \infty}\left\|Q x-T\left(t_{n}\right) Q x\right\| \leq(1+\eta(\mathcal{F}))\left\|x_{m}-Q x\right\|+D_{m} \rightarrow 0 \quad \text { as } m \rightarrow \infty .
$$

Hence $\lim _{n \rightarrow \infty} T\left(t_{n}\right) Q x=Q x$. Let $s \in G$. From the demicontinuity of $T(s)$, we obtain that $T(s) Q x=Q x$. One can see that $T(s) Q x=Q x$ for all $s \in G$. Thus, $T(s) Q x=Q x$ for all $x \in C$ and $s \in G$. Therefore, $Q$ is a retraction of $C$ onto $\operatorname{Fix}(\mathcal{F})$. 
Corollary 4.2 Let X be a uniformly Banach space with the Opial condition, $C$ a nonempty closed convex bounded subset of $X$ and $T: C \rightarrow C$ a demicontinuous asymptotically regular nearly Lipschitzian mapping such that $\eta_{\infty}(T):=\liminf _{n \rightarrow \infty} \eta\left(T^{n}\right)<\sqrt{\operatorname{WCS}(X)}$. Then $\operatorname{Fix}(T) \neq \emptyset$ and $\operatorname{Fix}(T)$ is a $\eta_{\infty}(T)$-Lipschitzian retract of $C$.

One sees from Theorem 4.1 that if $\eta(\mathcal{F})=1$, then $\operatorname{Fix}(\mathcal{F})$ is a nonexpansive retract of $C$. In the next section, we show that $\operatorname{Fix}(\mathscr{F})$ is a sunny nonexpansive retract of $C$ when $\mathcal{F}=\left\{T(t): t \in \mathbb{R}^{+}\right\}$a strongly continuous semigroup of asymptotically pseudo-contractive mappings (see Theorem 5.6).

\section{Common fixed-point sets as sunny nonexpansive retracts}

Let $C$ be a nonempty subset of a Banach space $X$ and $\mathcal{F}=\{T(t): t \in G\}$ a semigroup of mappings from $C$ into itself. A sequence $\left\{x_{n}\right\}$ in $C$ is said to an approximating fixed point sequence of $\mathcal{F}$ if $\lim _{n \rightarrow \infty}\left(x_{n}-T(t) x_{n}\right)=0$ for all $t \in G$. The family $\{I-T(t): t \in G\}$ is demiclosed at zero if $\left\{y_{n}\right\}$ is a sequence in $C$ weakly converging to $z \in C$ and $\lim _{n \rightarrow \infty}\left(x_{n}-\right.$ $\left.T(t) y_{n}\right)=0$ for all $t \in G$ imply $z=T(t) z$ for all $t \in G$. Following [18], we say that $\mathcal{F}$ has property (AA) if for every bounded set $\left\{x_{t}\right\}_{t \in G}$ in $C$, we have

$$
\lim _{t \rightarrow \infty}\left\|x_{t}-T(t) x_{t}\right\|=0 \quad \text { implies } \quad \lim _{t \rightarrow \infty}\left\|x_{t}-T(s) x_{t}\right\|=0 \quad \text { for all } s \in G .
$$

In [30], Schu introduced the concept of asymptotically pseudo-contractive mapping as follows:

Let $H$ be a real Hilbert space whose inner product and norm are denoted by $\langle\cdot, \cdot\rangle$ and $\|\cdot\|$ respectively. Let $C$ be a nonempty subset of $H$ and $T: C \rightarrow C$ a mapping. Then $T$ is called an asymptotically pseudo-contractive mapping if there exists a sequence $\left\{k_{n}\right\}$ in $[1, \infty)$ with $\lim _{n \rightarrow \infty} k_{n}=1$ such that

$$
\left\langle T^{n} x-T^{n} y, x-y\right\rangle \leq k_{n}\|x-y\|^{2} \quad \text { for all } x, y \in C \text { and } n \in \mathbb{N} .
$$

The class of asymptotically pseudo-contractive mappings contain properly the class of asymptotically nonexpansive mappings. The following example shows that a continuous asymptotically pseudo-contractive mapping is not necessarily asymptotically nonexpansive.

Example 5.1 Let $X=\mathbb{R}$ and $C=[0,1]$. Define $T: C \rightarrow C$ by

$$
T x=\left(1-x^{2 / 3}\right)^{3 / 2}, \quad x \in C .
$$

Note that $T$ is a pseudo-contractive mapping which is not Lipschitzian (see [31]). Since $T$ is not Lipschitzian, it is not asymptotically nonexpansive. It is shown in [30] that $T$ is an asymptotically pseudo-contractive mapping with sequence $\{1\}$.

Let $\mathcal{F}=\{T(t): t \in G\}$ be a semigroup of mappings from $C$ into itself. Then $\mathcal{F}$ is said to be pseudo-contractive if

$$
\langle T(t) x-T(t) y, x-y\rangle \leq\|x-y\|^{2} \quad \text { for all } x, y \in C \text { and } t \in G \text {. }
$$




\section{Remark 5.2}

(i) The semigroup $\mathcal{F}$ is pseudo-contractive if and only if the following holds:

$$
\|T(t) x-T(t) y\|^{2} \leq\|x-y\|^{2}+\|x-T(t) x-(y-T(t) y)\|^{2} \quad \text { for all } x, y \in C \text { and } t \in G \text {. }
$$

(ii) Every nonexpansive semigroup must be a continuously pseudo-contractive semigroup.

We say $\mathcal{F}$ is asymptotically pseudo-contractive if there exists a function $k(\cdot): G \rightarrow[1, \infty)$ with $\lim _{t \rightarrow \infty} k(t)=1$ such that

$$
\langle T(t) x-T(t) y, x-y\rangle \leq k(t)\|x-y\|^{2} \quad \text { for all } x, y \in C \text { and } t \in G \text {. }
$$

Example 5.3 Let $X=\mathbb{R}, b \in(0,1), C=[0,1]$ and $G=[0, \infty)$. For $t>0$, define $T(t): C \rightarrow C$ by

$$
T(t) x= \begin{cases}b^{t} x, & \text { if } x \in[0,1 / 2] \\ 0, & \text { if } x \in(1 / 2,0]\end{cases}
$$

and define

$$
T(0) x=x, \quad x \in C .
$$

Set $C_{1}:=[0,1 / 2]$ and $C_{2}:=(1 / 2,1]$. Note that

$$
\langle T(t) x-T(t) y, x-y\rangle=b^{t}|x-y|^{2} \leq|x-y|^{2} \quad \text { for all } x, y \in C_{1} \text { and } t>0
$$

and

$$
\langle T(t) x-T(t) y, x-y\rangle=0 \leq|x-y|^{2} \quad \text { for all } x, y \in C_{2} \text { and } t>0 .
$$

For $x \in C_{1}$ and $y \in C_{2}$, we have $x-y \leq 0$, and hence

$$
\langle T(t) x-T(t) y, x-y\rangle=\left(b^{t} x-0\right)(x-y) \leq 0 \leq|x-y|^{2} \quad \text { for all } t>0 .
$$

Thus,

$$
\left\langle T(t) x-T(t) y, x-y|\leq| x-\left.y\right|^{2} \quad \text { for all } x, y \in C \text { and } t \geq 0 .\right.
$$

Therefore, $\mathcal{F}=\{T(t): t \geq 0\}$ is an asymptotically pseudo-contractive semigroup with function $k=1$. Moreover, for each $t>0, T(t)$ is discontinuous at $x=1 / 2$ and hence $\mathcal{F}$ is not a Lipschitzian semigroup.

We begin with the following:

Theorem 5.4 (Demiclosedness Principle) Let $C$ be a nonempty closed convex bounded subset of a real Hilbert space $H$. Let $\mathcal{F}=\{T(t): t \in G\}$ be a strongly continuous semigroup of 
uniformly continuous nearly uniformly L-Lipschitzian asymptotically pseudo-contractive mappings from $C$ into itself. Then the family $\{I-T(t): t \in G\}$ is demiclosed at zero.

Proof Assume that $\left\{y_{n}\right\}$ is a sequence in $C$ weakly converging to $z$ and $\lim _{n \rightarrow \infty}\left(y_{n}-\right.$ $\left.T(s) y_{n}\right)=0$ for all $s \in G$. Let $t \in G$ with $t \neq 0$ and let $\left\{t_{m}\right\}$ be a sequence in $G$ defined by $t_{m}=m t$ for all $m \in \mathbb{N}$. Notice that $z \in C$. Fix $\alpha \in(0,1 /(1+L))$ and define

$$
z_{m}=(1-\alpha) z+\alpha T\left(t_{m}\right) z \quad \text { for all } m \in \mathbb{N} \text {. }
$$

Since $T$ is uniformly continuous, we have $\left\|y_{n}-T\left(t_{m}\right) z\right\| \rightarrow 0$ as $n \rightarrow \infty$ for fixed $m \in \mathbb{N}$. Indeed, for fixed $m \in \mathbb{N}$, we have

$$
\begin{gathered}
\left\|y_{n}-T\left(t_{m}\right) y_{n}\right\| \leq \\
+\left\|y_{n}-T(t) y_{n}\right\|+\left\|T(t) y_{n}-T(2 t) y_{n}\right\|+\cdots \\
+\left\|T((m-1) t) y_{n}-T(m t) y_{n}\right\|
\end{gathered}
$$

for all $n \in \mathbb{N}$. Since $\mathcal{F}$ is a uniformly continuous semigroup, it follows that $\lim _{n \rightarrow \infty} \| y_{n}-$ $T\left(t_{m}\right) y_{n} \|=0$ for each fixed $m \in \mathbb{N}$. Noticing that $\mathcal{F}$ is an asymptotically pseudocontractive semigroup, for fixed $m \in \mathbb{N}$, we have

$$
\begin{aligned}
\left\langle z-z_{m},\left(I-T\left(t_{m}\right)\right) z_{m}\right\rangle= & \left\langle z-y_{n},\left(I-T\left(t_{m}\right)\right) z_{m}\right\rangle+\left\langle y_{n}-z_{m},\left(I-T\left(t_{m}\right)\right) z_{m}\right\rangle \\
= & \left\langle z-y_{n},\left(I-T\left(t_{m}\right)\right) z_{m}\right\rangle \\
& +\left\langle y_{n}-z_{m},\left(I-T\left(t_{m}\right)\right) z_{m}-\left(I-T\left(t_{m}\right)\right) y_{n}\right\rangle \\
& +\left\langle y_{n}-z_{m},\left(I-T\left(t_{m}\right)\right) y_{n}\right\rangle \\
\leq & \left\langle z-y_{n},\left(I-T\left(t_{m}\right)\right) z_{m}\right\rangle+\left(k\left(t_{n}\right)-1\right)\left\|y_{n}-z_{m}\right\|^{2} \\
& +\left\langle y_{n}-z_{m},\left(I-T\left(t_{m}\right)\right) y_{n}\right\rangle .
\end{aligned}
$$

Since $y_{n} \rightarrow z$ and $\lim _{n \rightarrow \infty}\left\|y_{n}-T\left(t_{m}\right) y_{n}\right\|=0$, it follows from (5.1) that

$$
\left\langle z-z_{m},\left(I-T\left(t_{m}\right)\right) z_{m}\right\rangle \leq\left(k\left(t_{n}\right)-1\right) \operatorname{diam}(C) .
$$

Note that

$$
\begin{aligned}
\left\|z-T\left(t_{m}\right) z\right\|^{2} & =\left\langle z-T\left(t_{m}\right) z, z-T\left(t_{m}\right) z\right\rangle \\
& =\frac{1}{\alpha}\left\langle z-z_{m}, z-T\left(t_{m}\right) z\right\rangle \\
& =\frac{1}{\alpha}\left\langle z-z_{m},\left(I-T\left(t_{m}\right)\right) z-\left(I-T\left(t_{m}\right)\right) z_{m}+\left(I-T\left(t_{m}\right)\right) z_{m}\right\rangle \\
& =\frac{1}{\alpha}\left[\left\langle z-z_{m},\left(I-T\left(t_{m}\right)\right) z-\left(I-T\left(t_{m}\right)\right) z_{m}\right\rangle+\left\langle z-z_{m},\left(I-T\left(t_{m}\right)\right) z_{m}\right\rangle\right] \\
& \leq \frac{1}{\alpha}\left[\left\|z-z_{m}\right\|^{2}+\left\|z-z_{m}\right\|\left\|T\left(t_{m}\right) z-T\left(t_{m}\right) z_{m}\right\|+\left(k\left(t_{m}\right)-1\right) \operatorname{diam}(C)\right] \\
& \leq \frac{1}{\alpha}\left[\left\|z-z_{m}\right\|^{2}+L\left\|z-z_{m}\right\|\left(\left\|z-z_{m}\right\|+a\left(t_{m}\right)\right)+\left(k\left(t_{m}\right)-1\right) \operatorname{diam}(C)\right] \\
& \leq \frac{1}{\alpha}\left[\alpha^{2}(1+L)\left\|z-T\left(t_{m}\right) z\right\|^{2}+\left(a\left(t_{m}\right) L+k\left(t_{m}\right)-1\right) \operatorname{diam}(C)\right],
\end{aligned}
$$


which implies that

$$
\alpha[1-\alpha(1+L)]\left\|z-T\left(t_{m}\right) z\right\|^{2} \leq\left(a\left(t_{m}\right) L+k\left(t_{m}\right)-1\right) \operatorname{diam}(C) \quad \text { for all } m \in \mathbb{N} .
$$

Letting $m \rightarrow \infty$ in (5.2), we obtain that $T\left(t_{m}\right) z \rightarrow z$. It follows from the continuity of $T(t)$ that

$$
z=\lim _{m \rightarrow \infty} T\left(t+t_{m}\right) z=\lim _{m \rightarrow \infty} T(t) T\left(t_{m}\right) z=T(t) z
$$

Therefore, $z=T(t) z$ for all $t \in G$.

The following result extends the celebrated convergence theorem of Browder [32] and many results concerning Browder's convergence theorem to a semigroup of uniformly continuous nearly uniformly $L$-Lipschitzian asymptotically pseudo-contractive mappings.

Theorem 5.5 Let $C$ be a nonempty closed convex bounded subset of a real Hilbert space $H$ and $\mathcal{F}=\left\{T(t): t \in \mathbb{R}^{+}\right\}$a strongly continuous semigroup of uniformly continuous nearly uniformly L-Lipschitzian asymptotically pseudo-contractive mappings from $C$ into itself. Let $\left\{b_{n}\right\}$ be a sequence in $(0,1)$ and $\left\{t_{n}\right\}$ a sequence in $(0, \infty)$ such that $k\left(t_{n}\right)-1<b_{n}$ for all $n \in \mathbb{N}, \lim _{n \rightarrow \infty} b_{n}=\lim _{n \rightarrow \infty} \frac{k\left(t_{n}\right)-1}{b_{n}}=0$ and $\lim _{n \rightarrow \infty} t_{n}=\infty$. Then:

(a) There exists a sequence $\left\{y_{n}\right\}$ in $C$ defined by

$$
y_{n}=b_{n} u+\left(1-b_{n}\right) T\left(t_{n}\right) y_{n}, \quad n \in \mathbb{N} .
$$

(b) If $\mathcal{F}$ has property $(\mathcal{A})$, then $\operatorname{Fix}(\mathcal{F}) \neq \emptyset$ and $\left\{y_{n}\right\}$ converges strongly to $y^{*} \in \operatorname{Fix}(\mathcal{F})$ such that

$$
\left\langle y^{*}-u, y^{*}-v\right\rangle \leq 0 \quad \text { for all } v \in \operatorname{Fix}(\mathcal{F}) .
$$

Proof

(a) Let $\mathcal{F}=\left\{T(t): t \in \mathbb{R}^{+}\right\}$be a strongly continuous semigroup of asymptotically pseudocontractive mappings with a net $\{k(t): t \in(0, \infty)\}$. Set $\varrho_{n}:=\frac{k\left(t_{n}\right)-1}{b_{n}}$. Note $k\left(t_{n}\right)-1<b_{n}$ for all $n \in \mathbb{N}$, it follows that $\varrho_{n}<1 \leq k\left(t_{n}\right)$ and hence $\left(1-b_{n}\right) k\left(t_{n}\right)<1$ for all $n \in \mathbb{N}$. Then, for each $n \in \mathbb{N}$, the mapping $G_{n}: C \rightarrow C$ defined by

$$
G_{n} y:=b_{n} u+\left(1-b_{n}\right) T\left(t_{n}\right) y, \quad y \in C
$$

is continuous and strongly pseudo-contractive. Indeed, for $x, y$ in $C$, we have

$$
\begin{aligned}
\left\langle G_{n} x-G_{n} y, x-y\right\rangle & =\left(1-b_{n}\right)\left\langle T\left(t_{n}\right) x-T\left(t_{n}\right) y, x-y\right\rangle \\
& \leq\left(1-b_{n}\right) k\left(t_{n}\right)\|x-y\|^{2} .
\end{aligned}
$$

Therefore, by Lemma 2.1, there exists a sequence $\left\{y_{n}\right\}$ in $C$ described by (5.3).

(b) Assume that $\mathcal{F}$ has property (AA). From (5.3), we have $y_{n}-T\left(t_{n}\right) y_{n} \rightarrow 0$ as $n \rightarrow \infty$. The property (A) of $\mathcal{F}$ gives that $y_{n}-T(t) y_{n} \rightarrow 0$ as $n \rightarrow \infty$ for all $t>0$. Since $\left\{y_{n}\right\}$ is 
bounded, we can assume that a subsequence $\left\{y_{n_{i}}\right\}$ of $\left\{y_{n}\right\}$ such that $y_{n_{i}} \rightarrow z$ for some $z \in C$.

By Theorem 5.4, we have $z \in \operatorname{Fix}(\mathcal{F})$.

For $v \in \operatorname{Fix}(\mathcal{F})$, we have

$$
\begin{aligned}
\left\langle y_{n}-T\left(t_{n}\right) y_{n}, y_{n}-v\right\rangle & =\left\langle y_{n}-v+T\left(t_{n}\right) v-T\left(t_{n}\right) y_{n}, y_{n}-v\right\rangle \\
& \geq-\left(k\left(t_{n}\right)-1\right)\left\|y_{n}-v\right\|^{2} .
\end{aligned}
$$

From (5.3), we have

$$
\begin{aligned}
\left\langle y_{n}-u, y_{n}-v\right\rangle & =\left(1-b_{n}\right)\left\langle T\left(t_{n}\right) y_{n}-u, y_{n}-v\right\rangle \\
& =\left(1-b_{n}\right)\left\langle T\left(t_{n}\right) y_{n}-y_{n}+y_{n}-u, y_{n}-v\right\rangle,
\end{aligned}
$$

so it follows that

$$
\begin{aligned}
\left\langle y_{n}-u, y_{n}-v\right\rangle & \leq \frac{1-b_{n}}{b_{n}}\left\langle T\left(t_{n}\right) y_{n}-y_{n}, y_{n}-v\right\rangle \\
& \leq\left(1-b_{n}\right) \varrho_{n}\left\|y_{n}-v\right\|^{2} .
\end{aligned}
$$

Since $\lim _{n \rightarrow \infty} \varrho_{n}=0$ and $C$ is bounded, it follows from (5.5) that

$$
\limsup _{n \rightarrow \infty}\left\langle y_{n}-u, y_{n}-v\right\rangle \leq 0 \quad \text { for all } v \in \operatorname{Fix}(\mathcal{F})
$$

We claim that the set $\left\{y_{n}\right\}$ is sequentially compact. For $v \in \operatorname{Fix}(\mathcal{F})$, we have

$$
\begin{aligned}
\left\|y_{n}-v\right\|^{2} & =\left\langle b_{n}(u-v)+\left(1-b_{n}\right)\left(T\left(t_{n}\right) y_{n}-v\right), y_{n}-v\right\rangle \\
& \leq b_{n}\left\langle u-v, y_{n}-v\right\rangle+\left(1-b_{n}\right) k\left(t_{n}\right)\left\|y_{n}-v\right\|^{2},
\end{aligned}
$$

which implies that

$$
\left\|y_{n}-v\right\|^{2} \leq \frac{b_{n}}{1-\left(1-b_{n}\right) k\left(t_{n}\right)}\left\langle u-v, y_{n}-v\right\rangle .
$$

By the weak compactness of $C$, there exists a weakly convergent subsequence $\left\{y_{n_{i}}\right\} \subseteq\left\{y_{n}\right\}$. Suppose that $y_{n_{i}} \rightarrow y^{*} \in C$ as $i \rightarrow \infty$. Since $\left\{y_{n}\right\}$ is an approximating fixed point sequence of $\mathcal{F}$, we infer from Theorem 5.4 that $y^{\prime \prime} \in \operatorname{Fix}(\mathcal{F})$. In (5.7), interchange $v$ and $y^{*}$ to obtain that

$$
\left\|y_{n_{i}}-y^{*}\right\|^{2} \leq \frac{b_{n_{i}}}{1-\left(1-b_{n_{i}}\right) k\left(t_{n_{i}}\right)}\left\langle u-y^{*}, y_{n_{i}}-y^{*}\right\rangle .
$$

Since $y_{n_{i}} \rightarrow y^{*}$, we get that $y_{n_{i}} \rightarrow y^{*}$. Hence the set $\left\{y_{n}\right\}$ is sequentially compact.

Next, we show that $y_{n} \rightarrow y^{*}$. Suppose, for contradiction, that $\left\{y_{n_{j}}\right\}$ is another subsequence of $\left\{y_{n}\right\}$ such that $y_{n_{j}} \rightarrow z^{*} \neq y^{*}$. It is easy to see that $z^{*} \in \operatorname{Fix}(\mathcal{F})$. Observe that

$$
\begin{aligned}
\left|\left\langle y_{n}-u, y_{n}-z^{*}\right\rangle-\left\langle y^{*}-u, y^{*}-z^{*}\right\rangle\right| \leq & \left|\left\langle y_{n}-u, y_{n}-z^{*}\right\rangle-\left\langle y^{*}-u, y_{n}-z^{*}\right\rangle\right| \\
& +\left|\left\langle y^{*}-u, y_{n}-z^{*}\right\rangle-\left\langle y^{*}-u, y^{*}-z^{*}\right\rangle\right|
\end{aligned}
$$




$$
\begin{aligned}
\leq & \left\|y_{n}-u-\left(y^{*}-u\right)\right\|\left\|y_{n}-z^{*}\right\| \\
& +\left|\left\langle y^{*}-u, y_{n}-y^{*}\right\rangle\right| \text { for all } n \in \mathbb{N} .
\end{aligned}
$$

Since $y_{n_{i}} \rightarrow y^{*}$, we get

$$
\left\langle y_{n_{i}}-u, y_{n_{i}}-z^{*}\right\rangle \rightarrow\left\langle y^{*}-u, y^{*}-z^{*}\right\rangle
$$

From (5.6), we obtain

$$
\left\langle y^{*}-u, y^{*}-z^{*}\right\rangle \leq 0 \text {. }
$$

Similarly, we have

$$
\left\langle z^{*}-u, z^{*}-y^{*}\right\rangle \leq 0 \text {. }
$$

Adding inequalities (5.8) and (5.9) yields

$$
\left\langle y^{*}-z^{*}, y^{*}-z^{*}\right\rangle \leq 0
$$

a contradiction. In a similar way it can be shown that each cluster point of the sequence $\left\{y_{n}\right\}$ is equal to $y^{*}$. Therefore, the entire sequence $\left\{y_{n}\right\}$ converges strongly to $y^{*}$. It is easy to see, from (5.6), that the inequality (5.4) holds.

Theorem 5.6 Let $C$ be a nonempty closed convex bounded subset of a real Hilbert space $H$ and $\mathcal{F}=\left\{T(t): t \in \mathbb{R}^{+}\right\}$a strongly continuous semigroup of uniformly continuous nearly uniformly L-Lipschitzian asymptotically pseudo-contractive mappings from $C$ into itself. Suppose that $\mathcal{F}$ has property $(\mathcal{A})$. Then $\operatorname{Fix}(\mathcal{F}) \neq \emptyset$ and $\operatorname{Fix}(\mathcal{F})$ is a sunny nonexpansive retract of $C$.

Proof Assume that $\mathcal{F}=\left\{T(t): t \in \mathbb{R}^{+}\right\}$is a semigroup of asymptotically pseudo-contractive mappings from $C$ into itself with a function $k(\cdot): \mathbb{R}^{+} \rightarrow[1, \infty)$ with $\lim _{t \rightarrow \infty} k(t)=1$. Without loss of generality, we may assume that $\left\{b_{n}\right\}$ in $(0,1)$ and $\left\{t_{n}\right\}$ in $(0, \infty)$ such that $k\left(t_{n}\right)-1<b_{n}$ for all $n \in \mathbb{N}, \lim _{n \rightarrow \infty} b_{n}=\lim _{n \rightarrow \infty} \frac{k\left(t_{n}\right)-1}{b_{n}}=0$ and $\lim _{n \rightarrow \infty} t_{n}=\infty$. Then, for an arbitrarily fixed element $u \in C$, there exists a sequence $\left\{y_{n}\right\}$ in $C$ defined by (5.3). By Theorem 5.5(b), $\operatorname{Fix}(\mathcal{F}) \neq \emptyset$.

By Theorem 5.5(b), $\left\{y_{n}\right\}$ converges strongly to an element $y^{*} \in \operatorname{Fix}(\mathcal{F})$ such that the inequality (5.4) holds. Define a mapping $Q: C \rightarrow \operatorname{Fix}(\mathcal{F})$ by

$$
Q u=\lim _{n \rightarrow \infty} y_{n}, \quad u \in C .
$$

In view of (5.4), we have

$$
\langle Q u-u, Q u-v\rangle \leq 0 \quad \text { for all } u \in C \text { and } v \in \operatorname{Fix}(\mathcal{F}) .
$$

Therefore, by Lemma 2.2, we conclude that $Q$ is sunny nonexpansive. 


\section{Application}

Let $C$ be a nonempty convex subset of a real Hilbert space $H$ and $D$ a nonempty subset of $C$. For a nonlinear mapping $\mathcal{F}: C \rightarrow H$, the variational inequality problem $\operatorname{VIP}(\mathcal{F}, C)$ over $D$ is to find a point $x^{*} \in D$ such that

$$
\left\langle\mathcal{F} x^{*}, v-x^{*}\right\rangle \geq 0 \text { for all } v \in D \text {. }
$$

It is important to note that the theory of variational inequalities has played an important role in the study of many diverse disciplines, for example, partial differential equations, optimal control, optimization, mathematical programming, mechanics, finance, etc.; see, for example, $[33,34]$ and references therein.

We now turn our attention to dealing with the problem of the existence of solutions of $\operatorname{VIP}(C, \mathcal{F})$ by sunny nonexpansive retractions.

Following Wong, Sahu and Yao [35, Proposition 4.6], one can show that the variational inequality problem $\operatorname{VIP}(C, \mathcal{F})$ with $\mathcal{F}=I-f$ is equivalent to the fixed point problem. Indeed,

Proposition 6.1 Let $C$ be a nonempty convex subset of a smooth Banach space $X$ and $\mathcal{F}=\left\{T(t): t \in \mathbb{R}^{+}\right\}$a strongly continuous semigroup of mappings from $C$ into itself with $\operatorname{Fix}(\mathcal{F}) \neq \emptyset$. Let $f: C \rightarrow C$ be a mapping with $\mathcal{F}=I-f$ and let $Q$ be the sunny nonexpansive retraction from $C$ onto $\operatorname{Fix}(\mathcal{F})$. Then $x^{*}$ is a solution of variational inequality problem $\operatorname{VIP}(C, \mathcal{F})$ over $\operatorname{Fix}(\mathcal{F})$ if and only if $x^{\prime \prime}$ is a fixed point of $Q f$.

The following result improves the so-called viscosity approximation method which was first introduced by Moudafi [36] from nonexpansive mappings to a semigroup of pseudocontractive mappings.

Theorem 6.2 Let $C$ be a nonempty closed convex bounded subset of a real Hilbert space $H, f: C \rightarrow C$ a weakly contractive mapping with function $\psi$ and $\mathcal{F}=\left\{T(t): t \in \mathbb{R}^{+}\right\} a$ strongly continuous semigroup of uniformly continuous pseudo-contractive mappings from $C$ into itself. Suppose that $\mathcal{F}$ has property $(\mathcal{A})$ and $\mathcal{F}$ is nearly nonexpansive with function $a:[0, \infty) \rightarrow[0, \infty)$. Let $\left\{b_{n}\right\}$ be a sequence in $(0,1)$ and $\left\{t_{n}\right\}$ a sequence in $(0, \infty)$ such that $\lim _{n \rightarrow \infty} b_{n}=\lim _{n \rightarrow \infty} \frac{a\left(t_{n}\right)}{b_{n}}=0$ and $\lim _{n \rightarrow \infty} t_{n}=\infty$. Then, we have the following:

(a) The variational inequality problem $\operatorname{VIP}(C, I-f)$ over $\operatorname{Fix}(\mathcal{F})$ has a unique solution in $\operatorname{Fix}(\mathcal{F})$.

(b) There exists a sequence $\left\{y_{n}\right\}$ in $C$ defined by

$$
y_{n}=b_{n} f\left(y_{n}\right)+\left(1-b_{n}\right) T\left(t_{n}\right) y_{n}, \quad n \in \mathbb{N}
$$

such that $\left\{y_{n}\right\}$ converges strongly to the unique solution of the variational inequality problem $\operatorname{VIP}(C, I-f)$.

Proof

(a) By Theorem 5.6, there is a sunny nonexpansive retraction $Q$ from $C$ onto $\operatorname{Fix}(\mathcal{F})$. Since $Q f$ is a weakly contractive mapping from $C$ into itself, it follows from Rhoades [39, Theorem 1] that there exists a unique element $x^{*} \in C$ such that $x^{*}=Q f x^{*}$. Note $x^{*} \in C$ is 
an element of $\operatorname{Fix}(\mathcal{F})$. It follows from Proposition 6.1 that $x^{*}$ is the unique solution of the variational inequality problem $\operatorname{VIP}(C, I-f)$ over $\operatorname{Fix}(\mathcal{F})$.

(b) For each $n \in \mathbb{N}$, the mapping $F_{n}: C \rightarrow C$ defined by

$$
F_{n} y:=b_{n} f y+\left(1-b_{n}\right) T\left(t_{n}\right) y, \quad y \in C
$$

is continuous and strongly pseudo-contractive. In fact, for all $x, y \in C$ and $n \in \mathbb{N}$, we have

$$
\begin{aligned}
\left\langle F_{n} x-F_{n} y, x-y\right\rangle & =b_{n}\langle f x-f y, x-y\rangle+\left(1-b_{n}\right)\left\langle T\left(t_{n}\right) x-T\left(t_{n}\right) y, x-y\right\rangle \\
& \leq b_{n}\|f x-f y\|\|x-y\|+\left(1-b_{n}\right)\|x-y\|^{2} \\
& \leq b_{n}[\|x-y\|-\psi(\|x-y\|)]\|x-y\|+\left(1-b_{n}\right)\|x-y\|^{2} \\
& =\|x-y\|^{2}-b_{n} \psi(\|x-y\|)\|x-y\| .
\end{aligned}
$$

Hence each $F_{n}$ is continuous $b_{n} \psi$-strongly pseudo-contractive. Therefore, by [37, 38], there exists a sequence $\left\{y_{n}\right\}$ in $C$ described by (6.1). As in Theorem 5.5(a), we may define a sequence $\left\{z_{n}\right\}$ in $C$ by

$$
z_{n}=b_{n} f x^{*}+\left(1-b_{n}\right) T\left(t_{n}\right) z_{n} \quad \text { for all } n \in \mathbb{N} \text {. }
$$

By Theorem 5.5, we have that $z_{n} \rightarrow x^{*}=Q f x^{*}$. Observe that

$$
\begin{aligned}
\left\|y_{n}-z_{n}\right\| \leq & b_{n}\left\|f y_{n}-f x^{*}\right\|+\left(1-b_{n}\right)\left\|T\left(t_{n}\right) y_{n}-T\left(t_{n}\right) z_{n}\right\| \\
\leq & b_{n}\left(\left\|f y_{n}-f z_{n}\right\|+\left\|f z_{n}-f x^{*}\right\|\right)+\left(1-b_{n}\right)\left\|T\left(t_{n}\right) y_{n}-T\left(t_{n}\right) z_{n}\right\| \\
\leq & b_{n}\left(\left\|y_{n}-z_{n}\right\|-\psi\left(\left\|y_{n}-z_{n}\right\|\right)+\left\|z_{n}-x^{*}\right\|-\psi\left(\left\|z_{n}-x^{*}\right\|\right)\right) \\
& +\left(1-b_{n}\right)\left(\left\|y_{n}-z_{n}\right\|+a\left(t_{n}\right)\right) \\
\leq & \left\|y_{n}-z_{n}\right\|-b_{n} \psi\left(\left\|y_{n}-z_{n}\right\|\right)+b_{n}\left\|z_{n}-x^{*}\right\|+a\left(t_{n}\right) .
\end{aligned}
$$

It follows that

$$
\psi\left(\left\|y_{n}-z_{n}\right\|\right) \leq\left\|z_{n}-x^{*}\right\|+\frac{a\left(t_{n}\right)}{b_{n}} \quad \text { for all } n \in \mathbb{N} .
$$

Thus, $\left\|y_{n}-z_{n}\right\| \rightarrow 0$. Therefore, $x_{n} \rightarrow x^{*}=Q f x^{*}$.

\section{Competing interests}

The authors declare that they have no competing interests.

\section{Authors' contributions}

DRS designed of the study, performed the nonlinear analysis and also wrote the article. DO participated in the design of the study, carried out the materials and helped to check the manuscript. RA conceived of the study, participated in its design and also helped to draft the manuscript. All authors read and approved the final manuscript.

\section{Author details}

'Department of Mathematics, Banaras Hindu University, Varanasi, 221005, India. ²Department of Mathematics, Texas A\&M University, Kingsville, TX, USA. ${ }^{3}$ School of Mathematics, Statistics and Applied Mathematics, National University of Ireland Galway, Galway, Ireland. 


\section{References}

1. Benavides, TD, Ramirez, PL: Structure of the fixed point set and common fixed points of asymptotically nonexpansive mappings. Proc. Am. Math. Soc. 129, 3549-3557 (2001)

2. Goebel, K, Kirk, WA: A fixed point theorem for asymptotically nonexpansive mappings. Proc. Am. Math. Soc. 35, 171-174 (1972)

3. Agarwal, RP, O'Regan, D, Sahu, DR: Fixed Point Theory for Lipschitzian-type Mappings With Applications. Topological Fixed Point Theory and Its Applications, vol. 6. Springer, New York (2009)

4. Goebel, K, Reich, S: Uniform Convexity, Hyperbolic Geometry, and Nonexpansive Mappings. Dekker, New York (1984)

5. Kopecká, E, Reich, S: Nonexpansive retracts in Banach spaces. Banach Cent. Publ. 77, 161-174 (2007)

6. Reich, S: Extension problems for accretive sets in Banach spaces. J. Funct. Anal. 26, 378-395 (1977)

7. Bruck, RE Jr:: Properties of fixed-point sets of nonexpansive mappings in Banach spaces. Trans. Am. Math. Soc. 179 251-262 (1973)

8. Beauzamy, B: Projections contractantes dans les espaces de Banach. Bull. Sci. Math. 102, $43-47$ (1978)

9. Yao, JC, Zeng, LC: Fixed point theorems for asymptotically regular semigroups in metric spaces with uniform normal structure. J. Nonlinear Convex Anal. 8, 153-163 (2007)

10. Ceng, LC, Xu, HK, Yao, JC: Uniformly normal structure and uniformly Lipschitzian semigroups. Nonlinear Anal. 73, 3742-3750 (2010)

11. Belluce, LP, Kirk, WA: Fixed point theorems for certain classes of nonexpansive mappings. Proc. Am. Math. Soc. 20, 141-146 (1969)

12. Kirk, WA: Mappings of generalized contractive type. J. Math. Anal. Appl. 32, 567-572 (1970)

13. Kirk, WA: Asymptotic pointwise contractions. In: Plenary Lecture, the 8th International Conference on Fixed Point Theory and Its Applications, Chiang Mai University, Thailand, July 16-22 (2007)

14. Kirk, WA: Fixed points of asymptotic contractions. J. Math. Anal. Appl. 277, 645-650 (2003)

15. Kirk, WA, Xu, HK: Asymptotic pointwise contractions. Nonlinear Anal. 69, 4706-4712 (2008)

16. Casini, E, Maluta, E: Fixed points of uniformly Lipschitzian mappings in spaces with uniformly normal structure. Nonlinear Anal. 9, 103-108 (1985)

17. Lim, TC, Xu, HK: Fixed point theorems for asymptotically nonexpansive mappings. Nonlinear Anal. 22(11), 1345-1355 (1994)

18. Sahu, DR, Agarwal, RP, O'Regan, D: Structure of the fixed point set of asymptotically nonexpansive mappings in Banach spaces with weak uniformly normal structure. J. Anal. Appl. 17, 51-68 (2011)

19. Zegeye, $\mathrm{H}$, Shahzad, N: Strong convergence theorems for continuous semigroups of asymptotically nonexpansive mappings. Numer. Funct. Anal. Optim. 30(7-8), 833-848 (2009)

20. Chidume, CE, Zegeye, H: Strong convergence theorems for common fixed points of uniformly L-Lipschitzian pseudocontractive semi-groups. Appl. Anal. 86, 353-366 (2007)

21. Hester, A, Morales, CH: Semigroups generated by pseudo-contractive mappings under the Nagumo condition J. Differ. Equ. 245, 994-1013 (2008)

22. Bruck, RE, Kuczumow, T, Reich, S: Convergence of iterates of asymptotically nonexpansive mappings in Banach spaces with the uniform Opial property. Colloq. Math. LXV, 169-179 (1993)

23. Kirk, WA: Fixed point theorems for non-Lipschitzian mappings of asymptotically nonexpansive type. Isr. J. Math. 17, 339-346 (1974)

24. Sahu, DR: Fixed points of demicontinuous nearly Lipschitzian mappings in Banach spaces. Comment. Math. Univ. Carol. 46(4), 653-666 (2005)

25. Bynum, WL: Normal structure coefficient for Banach spaces. Pac. J. Math. 86, 427-436 (1980)

26. Benavides, TD, Acedo, GL, Xu, HK: Weak uniform normal structure and iterative fixed points of nonexpansive mappings. Colloq. Math. LXVIII, 17-23 (1995)

27. Gornicki, J: Fixed points of asymptotically regular semigroups in Banach spaces. Rend. Circ. Mat. Palermo 46, 89-118 (1997)

28. Sahu, DR, Zeqing, L, Kang, SM: Existence and approximation of fixed points of nonlinear mappings in spaces with weak uniform normal structure. Comput. Math. Appl. 64, 672-685 (2012)

29. Sahu, DR, Petruşel, A, Yao, JC: On fixed points of pointwise Lipschitzian type mappings. Fixed Point Theory (2012, accepted)

30. Schu, J: Iterative construction of fixed points of asymptotically nonexpansive mappings. J. Math. Anal. Appl. 158, 407-413 (1991)

31. Rhoades, BE: Comments on two fixed point iteration methods. J. Math. Anal. Appl. 56, 741-750 (1976)

32. Browder, FE: Convergence of approximants to fixed points of nonexpansive nonlinear mappings in Banach spaces. Arch. Ration. Mech. Anal. 24, 82-90 (1967)

33. Kinderlehrer, D, Stampacchia, G: An Introduction To Variational Inequalities and Their Applications. Academic Press, New York (1980)

34. Jaillet, P, Lamberton, D, Lapeyre, B: Variational inequalities and the pricing of American options. Acta Appl. Math. 21, 263-289 (1990)

35. Wong, NC, Sahu, DR, Yao, JC: Solving variational inequalities involving nonexpansive type mappings. Nonlinear Anal. 69, 4732-4753 (2008)

36. Moudafi, A: Viscosity approximation methods for fixed-points problems. J. Math. Anal. Appl. 241, 46-55 (2000)

37. Xiang, CH: Fixed point theorem for generalized F-pseudo-contractive mappings. Nonlinear Anal. 70, 277-279 (2009)

38. Morales, CH: Variational inequalities for $\Phi$-pseudo-contractive mappings. Nonlinear Anal. 75, 477-484 (2012)

39. Rhoades, BE: Some theorems on weakly contractive maps. Nonlinear Anal. 47, 2683-2693 (2001)

doi:10.1186/1687-1812-2012-163

Cite this article as: Sahu et al.: The structure of fixed-point sets of Lipschitzian type semigroups. Fixed Point Theory and Applications 2012 2012:163 